on cardiovascular outcome in these patients. Finally, no increase in cardiovascular risk was found in simple snorers.

Original article Marin JM et al. (2005) Long-term cardiovascular outcomes in men with obstructive sleep apnoea-hypopnoea with or without treatment with continuous positive airway pressure: an observational study. Lancet 365: 1046-1053

\section{Acute myocardial infarction: invasive versus medical treatment}

Despite evidence-based guidelines for the optimal treatment of patients with acute myocardial infarction (AMI), patterns of care vary considerably between different regions of the US. Stukel and colleagues asked whether more intensive treatment was associated with patients' clinical characteristics, and whether long-term survival was affected.

This 'natural experiment' included 158,831 elderly Medicare patients hospitalized for AMI in 566 coronary angiography service areas (CASAs). Patients were followed up for a mean period of 3.6 years. Although overall baseline AMI severity was similar across CASAs, the intensity of invasive treatment-defined as the proportion of eligible patients who underwent cardiac catheterization within 30 days-ranged from 29 to $93 \%$. Medical treatment intensity also varied greatly; the rate of prescription of $\beta$-blockers to eligible patients at discharge ranged from 5 to $93 \%$.

Survival at 7 years was $6.2 \%$ higher within CASAs providing the highest rates of intensive treatment than in those providing the lowest rates. In areas with the highest levels of $\beta$-blocker prescriptions, however, increased invasive treatment did not significantly improve survival. Importantly, younger, lower-risk patients were more likely to receive intensive treatment, despite the fact that older, higher-risk patients were more likely to benefit from these therapies. This 'treatment-risk paradox' has been shown previously and might reflect concerns about complications, or poor understanding of benefit-harm trade-offs.

In summary, dramatic variations in the provision of intensive treatment for AMI persist across different regions of the US. These do not appear to be related to patient characteristics and are associated with differences in survival. Because invasive treatment might not offer a survival benefit compared with optimal medical management, Stukel et al. call for large-scale evaluations of the long-term effectiveness of invasive cardiac technology at the population level.

Original article Stukel TA et al. (2005) Long-term outcomes of regional variations in intensity of invasive vs medical management of Medicare patients with acute myocardial infarction. JAMA 293: 1329-1337

\section{Prognostic value of BNP in heart failure}

\section{EBM}

B-type natriuretic peptide (BNP) and its precursor, $\mathrm{N}$-terminal pro-brain BNP (NT-proBNP) have been extensively studied for their predictive value in patients with heart failure. A systematic review now reveals that BNP is a strong prognostic indicator of cardiac risk in all patients, including those not diagnosed with heart failure by standard methods.

Doust and colleagues analyzed the results from 24 published studies that assessed the relationship between BNP and the risk of death or cardiovascular events. Of these, 19 included heart failure patients and 5 were based on asymptomatic individuals. Some of the studies used dichotomous measures of BNP, some used a continuous measure, and others used a change in BNP over time.

The results showed that each $100 \mathrm{pg} / \mathrm{ml}$ increase in BNP was related to a $35 \%$ increase in the relative risk of death among heart failure patients. In 23 out of 35 multivariate models, BNP or NT-proBNP were the strongest predictors of survival, cardiac death, readmission, or cardiac events. In nine of these models, BNP or NT-proBNP were the only variables that were significantly associated with prognosis. In addition, the risk of death among asymptomatic patients with raised BNP values (above approximately $20 \mathrm{pg} / \mathrm{ml}$ ) was twice that of patients with lower values.

Doust et al. acknowledge that reviews of this type have limitations, including the possibility of publication bias; nevertheless, the study shows that BNP is a strong prognostic indicator in heart failure, irrespective of disease stage. Furthermore, BNP appears to be 